\title{
The Application of Rich Media Technology in Digital Learning Terminal
}

\author{
Wu Yafen, Liao Chunhua, Nie Bin, Yang Qin \\ Research Department, Jiangxi University of Traditional Chinese Medicine \\ Jiangxi Nanchang, China 330004
}

\begin{abstract}
Keywords: Digital learning terminal; Rich media technology; Digital learning; Rich Internet application technology
\end{abstract}

\begin{abstract}
At present, digital learning terminal has larger impact on people's lives and students learning, and is likely to become more mature in a short period of time in the future. New digital terminal is built on the basis of reading. It is a dedicated terminal which is interactive, relevance, openness and has rich media features. It can help students to start meaningful learning. [1] Through the application and analysis of rich media and rich Internet and compared with multimedia technology features and digital terminal learning research, rich media and rich Internet technology can explore the terminals of digital learning, create a new type of digital learning environment, and further help and improve students' learning enthusiasm and initiative.

In people's life and learning, electronic products such as smart phones and tablet computers are widely used as terminal equipment. Gradually mobile reading and extensive electronic reading form becomes an important part in people's lives. E-books, with the fast development of electronic technology, get new developments. Digital textbooks, as special electronic books in education teaching, earn widespread interest and attention. Departments of Education put forward the corresponding requirements for digital education informatization in 2013, which includes: fully launch second generation People's Education Press digital textbooks, realize the development of digital products, and ensure to introduce scientific applicability product in the autumn of 2013. Deputy Minister of Departments of Education in our country put forward that we should insist on innovation and driving mechanism, fully implement the application in teaching and fully realize normalization and extensive application in Anhui province education information research. Modern teaching reform needs to rely on the innovation of digital teaching technology to truly achieve teaching wisdom and ascend the quality of teaching. It has become the inevitable development trend to apply digital teaching facilities in education and teaching, especially in elementary education class. [2] Although people make digital textbooks as the focus of the study, currently people have no unified understanding of digital teaching material, and issues such as the content design and application of digital textbook are still in the preliminary study stage.
\end{abstract}

\section{RICH MEDIA TECHNOLOGY APPLICATION}

\section{A. The characteristics of rich media technology}

Rich media mainly refers to the use of digital interactive media category. The technology is able to be downloaded and embedded in web pages. Rich media is to watch or offline use in Real player of Real Network and QuickTim of Apple. Driving is the most obvious characteristic of the technology and driving mechanism maintaining time is longer and can realize the indirect corresponding to the user operation.

After the analysis of state information technology data in University of Washington, it is known that rich media is not a very detailed form of Internet media and it refers to animation, sound, Flash, text and programming language embedded into JAVE. Rich media has the features: digital, interactive, free deployment in a web page, alone download, offline download, and can realize one-time download. But the number of using times is more common and it has a dynamic driving mechanism. It can fully realize user's synchronous operation and can realize cross-platform operation. It can support lightweight widgets. One of the most significant is UI show, a deeper level of user interaction, corresponding to dynamic drive and integrates with convenient deployment, 
desktop applications and network. [3]

\section{B. Rich Internet applications}

Rich Internet application is one of Web application types, which has multiple desktop application characteristics, browses on the plug-in and achieves a distributed system virtual machine, etc. Rich Internet application belongs to a new type of rich media network application technology. This technology can realize network terminal applications, and it has high similarity with C/S architecture, so it is more convenient in desktop application deployment and able to actualize network application client program. Rich Internet is regarded as a lightweight user operation interface. Because of this, it can be more adapted to low performance interrupt, and can better realize interface enhancement than using HTML. The reaction is more flexible and it has the characteristics of visualization and interaction. Rich Internet can provide interactive interface for message identification and format organization, and provide users with common interface. For example, it can be applied in WEB2.0 environment implementation, web page drag-and-drop, and in the process of refreshing Forms and offline operation etc.

Standing on the point of technical features, rich Internet engine can provide the technical support for timely response to the system, data exchange and rich user graphic interface. Rich Internet can allow SVG usage or other technologies graphics building, and can offer according data changes for whole activities animation. Its strength is in that data can be cached in the Client, and can be back and forth between the servers based on HTML at a faster corresponding speed. In mobile terminal equipment, the system can be designed as offline mode for a short period of time and implement sustainability. To jump out of the browser's limit, the interface can realize richer and more beautiful. Strongly ascend interface interaction ability and it is also a kind of network application. It has faster changes and deployment capabilities, and a strong dynamics in ActionScript script modification.

The goal of rich Internet is mainly to fully apply WEB in terminal desktop, fully fuse desktop and WEB, apply WEB, and integrate browser and WEB. The relevant scholars believe that the further development of rich Internet fully displays the coming of desktop rich Internet era.

\section{RICH MEDIA TECHNOLOGY APPLICATION IN DIGITAL LEARNING TERMINAL}

\section{A. Current digital terminal application}

At present, digital terminal products applications in learning in the market are mainly electronic reading products like E-book, mobile terminals installed electronic course ware learning process, etc., and students dedicated electronic textbooks and electronic schoolbag, etc. There is a wide variety with different characteristics. In the continuous development of learning concept, many products have to meet the digital learning needs.

E-book is an electronic reading terminal and the main focus is content show in the pursuit of a more lifelike paper effect, and fully provides learning resources. For example, Amazon's kindleDX can fully realize the effect of newspaper and try to establish a multifaceted digital publishing content. At present, all kinds of digital learning products constantly appear but they are mostly in the form of e-books, and still stay on the basis of E-link technology or mainly use Flash technology to produce a good electronic course ware. [4] General electronic textbooks are only the digital implementation of textbook content and did not achieve a real shift of digital textbooks.

Electronic course ware mainly takes learning theory as instruction, learning objectives as design aim, and fully reflects some strategies in learning and detailed teaching content. Electronic classes perform the corresponding user operations and further control the user's behavior for some feedback and control. Electronic course ware content is stable and will not implement corresponding adjustment by reader's feedback. All of the content is developed and designed ahead of schedule, so learning course ware resources have certain characteristics, such as closeness and reservation, no relevance and openness. This causes the difficulties of learning process and time cannot achieve consistent with the difference in learner's ability, and both cannot undertake adaptability adjustment.

B. Rich media technology application in digital learning terminal

1. Silverlight and Air technology

At present, there are two companies in the world make rich media technology research and 
development and earn a wide range of attention of people. The two companies are respectively Microsoft's Silverlight and the technology can realize RIA on the browser; the other is Adobe AIR, which represents desktop AIR. But the two companies' technologies are almost at the same level and gradually realize mutual fusion. RIA based on the browser commits to further enrichment of the desktop, and desktop lightweight AIR hopes to further strengthen browser's network features, and eventually becomes very effective application in the process of network interaction. [5]

RIA desktop and other types of terminal equipment technology are to achieve AIR visualization, and the goal will bring WEB into the next step. About the understanding of AdobeAIR, Adobe's vice President described: join in the use of Google, tell Web applications, and tell you before the storm. If then you close your browser, Google map can't continue to talk with you, but AdobeAIR becomes the middle part to connect the Internet with desktop. Even if you close the browser, it's still stored in the computer. Achieve the client's notice in Web application, and if a hurricane is coming, it will jump out to you in time.

Silverlight is a cross-platform type, which supports a variety of rich media technology, and is the concrete form to realize RIA, just like loading Flash technology in the browser; it can carry more browser content. Silverlight becomes Web desktop dynamic content creation, flexibly displays the existing resources and search engines and resources.

2. Android, widgets, and OMS

Both Silverlight and AIR are a kind of rich media technology implement system and need corresponding operating system in actual application. There are Windows series, Apple OS and Android, etc. on PC. This paper focuses on the support for digital and digital learning terminal implementation and the support for openness and interactivity. To this, the main study objects become smart phone, tablet, PAD, etc. There are a lot of mobile terminal operating systems, but at present the most popular is Apple IPAD, I phone system and Google Android.

At present, China mobile has started to develop OMS system with $3 \mathrm{~d}$ function on the basis of Android system. In addition to standard mobile, it already has 3 d type flip effect, and at the same time develops many applications. There are Chinese service menu, music, navigation and email, etc. in OMS system, which provides a good demonstration for the development of education field digital learning terminal system and one of the most distinctive is that Android system implemented support for widgets since version 1.5.

Widgets can be translated into micro parts, which is a very small piece existing in HTML Web for code execution, specifically including video, news, games, etc. In Widget, there are DHTML, JavaScript, etc. Widget application is mainly on Widget engine. Its deployment and running maintain independent state, which fully realizes user's Internet experience and ensures the timeliness of useful advice, information, and data.

\section{SUMMARY}

In conclusion, current social digital learning terminal standards and match degree between actual technical developments are very low, but rich media technology has gradually been appreciated. In the near future, rich media technology can get international and national standards with consistency to electronic reading, textbooks and studies.

\section{REFERENCE}

[1] Fu Wei. Rich media technology application and exploration in digital learning terminal [J]. Journal of Distance Education, 2011, 95:95-102.

[2] Huang Ronghuai, Yang Junfeng, Hu Yongbin. From digital learning environment to wisdom learning environment---- learning environment change and trend [J]. Open Education Research, 2012,01:75-84.

[3] Gu Xiaoqing, Fu Wei, Qi Guichao. Reading and study connection: electronic textbooks information model design [J]. Journal of East China Normal University (Natural Science Edition), 2012, 11:81-90. 
[4] Yu Xiqing. Rich media technology research and application in mobile social client [D]. Graduate School of Chinese Academy of Sciences (Shenyang Institute of Computing Technology), 2014.

[5] Huang Ruhua, Li Yingzi. Rich media material acquisition characteristics in MOOC----Information literacy classes for example [J]. Journal of Library and Intelligence, 2014:8 to 13. 